\title{
Les séries de puissances dont les restes ont seulement des zéros non-positifs
}

\section{Iossif Vladimirovich OSTROVSKII}

Department of Mathematics, Bilkent Universily, 06533 Bilkent, Ankara, Turkey and Verkin Institute for Low Temperature Physics and Engineering, 310164 Kharkov, Ukraine.

F-mail : iossif@fen.hilkent.edu.tr,ostrovskii@ilt.kharkov.ıa

Résumé. Supposons que le $n$-ième reste de la série de puissances d'une fonction entière $f$ a seulement des zéros non-positifs pour tout $n$ assez grand. Alors

$$
\log M(r, f) \leq \frac{1}{2 \log 2}(\log r)^{2}+O(\log r), \quad r \rightarrow \infty .
$$

Cette estimation est proche d'être optimale.

\section{Power series having tails with only real nonpositive zeroes}

Abstract. If for all sufficiently large $n$ the $n^{\text {th }}$ tail of the power series of an entire function $f$ has only real nonpositive zeroes, then

$$
\log M(r, f) \leq \frac{1}{2 \log 2}(\log r)^{2}+O(\log r), \quad r \rightarrow \infty .
$$

This bound is close to the best possible.

\section{Abridged English Version}

The Taylor series of an entire function $f(z)$ can be decomposed in the form of a partial sum of order $n, s_{n}(z)$, and a remainder, $t_{n}(z)$. Starting with Pólya, several authors studied the asymptotic behaviour of $M(r, f)\left(=\sup _{|z|=r}|f(z)|\right)$, under different assumptions on the localization of the zeroes of the partial sums $s_{n}(z)$. Here we assume that all zeroes of all remainders $t_{n}(z)$ are carried by the half real line $(-\infty, 0]$, and we prove

$$
\log M(r, f) \leq \frac{1}{2 \log 2}(\log r)^{2}+O(\log r), \quad r \rightarrow \infty
$$

(main theorem).

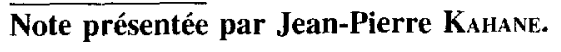




\section{V. Ostrovskii}

The proof of (1.6) relies on two lemmas of independent interest. Lemma 1 gives an estimate for $|f(z)|$, namely $(2.1)$, when $z$ belongs to an angle $A_{\alpha, \beta}(=\{z: \alpha<\arg z<\beta\})$ which contains only a finite number of zeroes of three different remainders $t_{n_{1}}(z), t_{n_{2}}(z)$, and $t_{n_{3}}(z)$. Lemma 2 gives an estimate for $M(r, f)$, namely $(2.3)$, assuming that all zeroes of $t_{n_{1}}(z) t_{n_{2}}(z) t_{n_{3}}(z)$, cxcept a finite number, lie on a system of half lines starting from 0. Lemma 2 leads to (1.6), via the Hadamard factorization theorem and estimates for the Taylor cocfficients of $f(z)$.

The order of magnitude of the second member of (1.6) is best possible, as the example

$$
f(z)=\sum_{k=0}^{\infty} 2^{-k^{2}} z^{k}
$$

shows. In that case

$$
\log M(r, f)=\frac{1}{4 \log 2}(\log r)^{2}+O(\log r), \quad r \rightarrow \infty
$$

\section{Introduction}

En 1913 G. Pólya (voir [10]) a démontré le théorème suivant :

Soit

$$
f(z)=\sum_{k=0}^{\infty} a_{k} z^{k}
$$

une série de puissances formelle. Supposons que, pour tout $n$ assez grand les sommes partielles

$$
s_{n}(z)=\sum_{k=0}^{n} a_{k} z^{k}
$$

ont seulement des zéros non-positifs. Alors la série est convergente dans tout le plan et

$$
\log M(r, f)=O\left((\log r)^{2}\right), \quad r \rightarrow \infty,
$$

où $M(r, f)=\max \{|f(z)|:|z|=r\}$.

Ultérieurement, les séries de puissances formelles, avec des restrictions pour les arguments des zéros de leurs sommes partielles, ont été profondément étudiées par beaucoup de mathématiciens (voir les références dans [4] et [5]). Le résultat final a été obtenu par T. Ganelius (voir [5]) en 1963 :

Soit (1.1) une série de puissances formelle. Admettons qu'une valeur positive $\gamma$ et une suite de nombres réels $\left\{\alpha_{k}\right\}_{k=0}^{\infty}$ existent telles que pour tout $n$ assez grand l'angle $\left\{z: \alpha_{n}<\arg z<\alpha_{n}+\gamma\right\}$ ne contient pas de zéro de sommes partielles (1.2). Alors (1.1) est convergente dans tout le plan et (1.3) a lieu.

Supposons maintenant d'avance que la série (1.1) est convergente dans tout le plan et considérons ses restes

$$
t_{n}(z)=\sum_{k=n+1}^{\infty} a_{k} z^{k} . \quad n=0,1,2, \ldots
$$


Le problème suivant semble intéressant : existe-t-il un analogue du théorème de Ganelius pour les restes?

Dans la présente Note un analogue est obtenu, mais uniquement pour le théorème de G. Pólya :

THÉORÈME. - Soit $f$ une fonction entière (1.1). Supposons, que pour tout $n$ assez grand, les restes (1.5) ont seulement des zéros non-positifs. Alors

$$
\log M(r, f) \leq \frac{1}{2 \log 2}(\log r)^{2}+O(\log r), \quad r \rightarrow \infty
$$

Dans un certain sens l'estimation (1.6) est proche d'être optimale :

Exemple. - Considérons la fonction

$$
f(z)=\sum_{k=0}^{\infty} 2^{-k^{2}} z^{k} .
$$

On sait (voir [11], Part V, Ch.3, no. 176) que tous les zéros de la fonction $f$ sont négatifs. Comme $l_{n}(z)=2^{-(n+1)^{2}} z^{n+1} f\left(2^{-2(n+1)} z\right)$, tous les zéros des restes $t_{n}(z)$ sont non-positifs. Des calculs standard montrent que

$$
\log M(r, f)=\frac{1}{4 \log 2}(\log r)^{2}+O(\log r), \quad r \rightarrow \infty .
$$

Notons que les zéros des restes de certaines séries de puissances concrètes (en particulier exponentielles) ont été étudiés par un certain nombre d'auteurs (voir [3], [7], [1] et [12]). Dans [2] a été étudié le comportement des modules des zéros des restes des séries de puissances générales. Notre théorème concerne le comportement des arguments des zéros des restes des séries de puissances générales entières; nous n'avons pas trouvé ce type de résultats dans la littérature.

\section{Les résultats préliminaires}

I.fmme 1. - Soit (1.1) une fonction entière. Supposons qu'il existe un angle

$$
A_{\alpha, \beta}=\{z: \alpha<\arg z<\beta\}, \quad \beta \leq \alpha+2 \pi
$$

et trois restes deux à deux distincts $t_{n_{1}}(z), t_{n_{2}}(z), t_{n_{3}}(z)$ tels que $A_{\alpha, \beta}$ ne contient qu'un nombre fini des zéros de la fonction $t_{n_{1}}(z) t_{n_{2}}(z) t_{n_{3}}(z)$. Alors

$$
\log \left|f\left(r e^{i \theta}\right)\right| \leq C \frac{r^{\pi / \gamma}}{\sin [\pi(\theta-\alpha) / \gamma]} \log \frac{r^{\pi / \gamma}}{\sin [\pi(\theta-\alpha) / \gamma]}, \quad r \geq 2, \quad \alpha<\theta<\beta,
$$

où $C$ est une constante positive et $\gamma=\beta-\alpha$.

Démonstration. - Sans perte de généralité on peut considérer l'angle $A_{-\gamma / 2, \gamma / 2}, 0<\gamma \leq 2 \pi$. En utilisant la notation (1.2), introduisons la fonction méromorphe

$$
q(z)=\frac{f(z)-s_{n_{1}}(z)}{f(z)-s_{n_{2}}(z)} \cdot \frac{s_{n_{3}}(z)-s_{n_{2}}(z)}{s_{n_{3}}(z)-s_{n_{1}}(z)} .
$$




\section{V. Ostrovskii}

Il suit des conditions du lemme que la fonction $q(z)$ prend au plus un nombre fini de fois les valeurs 0,1 et $\infty$ dans $A_{-\gamma / 2, \gamma / 2}$. Soit

$$
\zeta=\left(z^{\pi / \gamma}-1\right) /\left(z^{\pi / \gamma}+1\right)
$$

une application conforme de l'angle $A_{-\gamma / 2, \gamma / 2}$ sur le cercle unité $\Delta=\{|\zeta|<1\}$. La fonction $q(z(\zeta))$ est méromorphe dans $\Delta$ et y prend au plus un nombre fini de fois les valeurs 0,1 et $\infty$. Par un théorème de Nevanlinna (voir [9], Ch. X, $\mathbf{n}^{\circ} 2.7$, p. 271),

$$
T(r, q(z(\zeta)))=O[\log (1 /(1-r))], \quad r \rightarrow 1,
$$

où $T$ est la caractéristique de Nevanlinna de la fonction $q(z(\zeta))$. La fonction $f(z(\zeta))$ est homographique par rapport à $q(z(\zeta))$ avec des coefficients qui sont des polynômes en $z(\zeta)$. En utilisant les propriétés de la caractéristique de Nevanlinna (voir [9], Ch. VI, n 2.5, p. 170-171) et en tenant compte du fait que la caractéristique de Nevanlinna de la fonction $z(\zeta)$ (voir [9], Ch. VII, $\mathrm{n}^{\circ}$ 5.1, p. 209) est bornée, on obtient

$$
T(r, f(z(\zeta)))=O[\log (1 /(1-r))], \quad r \rightarrow 1 .
$$

Par une inégalité standard (voir [9], Ch. VIII, $\mathrm{n}^{\circ} 1.3$, p. 216), on a :

$$
\log M(r, f(z(\zeta))) \leq \frac{4}{1-r} T\left(\frac{1+r}{2}, f(z(\zeta))\right)=O\left(\frac{1}{1-r} \log \frac{1}{1-r}\right), \quad r \rightarrow 1,
$$

qui est équivalente à l'estimation

$$
\log |f(z(\zeta))| \leq\left[C_{1} /(1-|\zeta|)\right][\log (2 /(1-|\zeta|))], \quad|\zeta|<1, \quad C_{1}=\text { const }>0 .
$$

En notant que (2.2) implique

$$
1 /(1-|\zeta|) \leq\left(|z|^{\pi / \gamma}\right) / \cos [(\pi \arg z) / \gamma], \quad|z| \geq 1, \quad|\arg z|<\gamma / 2,
$$

on obtient (2.1) pour $\beta=-\alpha=\gamma / 2$.

LemMe 2. - Soit $f(z)$ une fonction entière (1.1). Supposons qu'il existe un système fini de rayons

$$
D=D\left(\alpha_{1}, \alpha_{2}, \ldots, \alpha_{n}\right)=\bigcup_{j=1}^{n}\left\{z: \arg z=\alpha_{j}\right\}, \quad \alpha_{1}<\alpha_{2}<\cdots<\alpha_{n} \leq \alpha_{n+1}=\alpha_{1}+2 \pi,
$$

et trois restes deux à deux différents $t_{n_{1}}(z), t_{n_{2}}(z), t_{n_{3}}(z)$ tels que tous les zéros de la fonction $t_{n_{1}}(z) t_{n_{2}}(z) t_{n_{3}}(z)$, à l'exception au plus d'un nombre fini, se trouvent sur le système $D$. Alors

$$
\log M(r, f)=O\left(r^{\pi / \gamma} \log r\right), \quad r \rightarrow \infty,
$$

où

$$
\gamma=\min \left\{\alpha_{j+1}-\alpha_{j}: 1 \leq j \leq n\right\} .
$$

Démonstration. - En appliquant le lemme 1 pour chaque angle $A_{\iota_{j}, \alpha_{j+1}}, j=1,2, \ldots, n$, on obtient pour $j=1,2, \ldots$ :

$$
\log \left|f\left(r e^{i \theta}\right)\right| \leq C \frac{r^{\pi / \gamma_{j}}}{\sin \left[\pi\left(\theta-\alpha_{j}\right) / \gamma_{j}\right]} \log \frac{r^{\pi / \gamma_{j}}}{\sin \left[\pi(\theta-\alpha) / \gamma_{j}\right]}, \quad r \geq 2, \alpha_{j}<\theta<\alpha_{j+1},
$$


où $C$ est une constante positive et $\gamma_{j}=\alpha_{j+1}-\alpha_{j}, j=1,2, \ldots, n$. Soit $q$ et $\delta$ des nombres fixés tels que $q>\pi / \gamma, 0<\delta<\gamma / 2$. Alors il résulte de (2.4) que (avec une constante $C$ plus grande)

$$
\log \left|f\left(r e^{i \theta}\right)\right| \leq C \frac{r^{q}}{\left|\sin \left(\theta-\alpha_{j}\right)\right|}, \quad r \geq 1, \quad\left|\theta-\alpha_{j}\right| \leq \delta, \quad j=1,2, \ldots, n .
$$

En appliquant le log log-théorème de Sjöberg-Levinson-Domar (voir par exemple [8], p. 376) pour chaque angle $A_{\alpha_{j}-\delta, \alpha_{j}+\varepsilon}$, on obtient :

$$
\log \left|f\left(r e^{i \theta}\right)\right| \leq C_{1} r^{q}, \quad r \geq 1, \quad\left|\theta-\alpha_{j}\right| \leq \delta / 2, \quad j=1,2, \ldots, n, \quad C_{1}=\text { const }>0 .
$$

On peut supposer que $\delta<\pi / q$. En utilisant (2.4) et en appliquant le principe de Phragmén-Lindelöf pour chaque angle $A_{\alpha_{j}-\delta / 2, \alpha_{j}+\delta / 2}$, on obtient (2.3).

Remarque. - L'estimation (2.3) peut être améliorée de la façon suivante :

$$
\log M(r, f)=O\left(r^{\pi / \gamma}\right), \quad r \rightarrow \infty
$$

On peut le démontrer en utilisant la théorie de Nevanlinna des fonctions méromorphes dans un angle (voir [12], Ch. 3, §3) et en prenant en considération que la fonction $\int(z)$ a un ordre fini par le lemme 2. Nous n'utilisons pas cette remarque ci-dessous.

\section{La démonstration du théorème}

Soit $f(z)$ une fonction entière (1.1) qui satisfait les conditions du théorème. En appliquant le lemme 2 pour le système composé du rayon unique $\{z: \arg z=\pi\}$ (pour ce cas $\gamma=2 \pi$ ), on obtient l'estimation

$$
\log M(r, f)=O(\sqrt{r} \log r), \quad r \rightarrow \infty
$$

Soit $m$ un nombre entier tel que tous les restes $t_{n}(z), n \geq m$, ont seulement des zéros non-positifs et, outre cela, le premier coefficient de $t_{m}(z)$ (c'est-à-dire $a_{m+1}$ ) est non-nul. L'estimation (3.1) montre que $t_{m}(z)$ est une fonction entière d'ordre plus petit que 1 , c'est pourquoi par le théorème de factorisation d'Hadamard, on a :

$$
\frac{1}{a_{m+1}} t_{m}(z) z^{-m-1}=\sum_{k=0}^{\infty} \frac{a_{k+m+1}}{a_{m+1}} z^{k}=\prod_{j=1}^{\infty}\left(1+\frac{z}{\lambda_{j}^{(m)}}\right),
$$

où $\lambda_{j}^{(m)}>0(j=1,2, \ldots), \sum_{j=1}^{\infty} 1 / \lambda_{j}^{(m)}<\infty$. Comme tous les coefficients de Taylor du produit infini dans (3.2) sonl évidemment positifs, on voit que $a_{n+1} \neq 0$ pour $n \geq m$ ct, outre cela, (3.2) reste vrai si on remplace $a_{k+m+1} / a_{m+1}$ par $\left|a_{k+m+1} / a_{m+1}\right|$ et ensuite $m$ par le nombre arbitraire $n \geq m$. Par la relation (3.2) modifiée de cette façon, on obtient:

$$
\left|\frac{a_{n+2}}{a_{n+1}}\right|^{2}-2\left|\frac{a_{n+3}}{a_{n+1}}\right|=\sum_{j-1}^{\infty}\left(\frac{1}{\lambda_{j}^{(n)}}\right)^{2}>0, \quad n=m, m+1, \ldots
$$

Cette inégalité peut être écrite comme

$$
\left|a_{n+3} / a_{n+2}\right|<(1 / 2)\left|a_{n+2} / a_{n+1}\right|, \quad n=m, m+1, \ldots
$$




\section{V. Ostrovskii}

Par induction on obtient :

$$
\left|a_{n+1} / a_{n}\right|<(1 / 2)^{n-m-1}\left|a_{m+2} / a_{m+1}\right|, \quad n=m+1, m+2, \ldots,
$$

et ensuite

$$
\left|a_{n}\right|<2^{-n^{2} / 2} D^{n+1}, \quad n=m+1, m+2, \ldots,
$$

où $D$ ne dépend pas de $n$. On obtient (1.6) à partir de la dernière inégalité, par des calculs standard.

Remerciements. Je remercie le Dr. C. Y. Yildirim à l'Université Bilkent, à Ankara, qui a attiré mon attention sur l'étude de la distribution des zéros des restes des séries de puissances et m'a indiqué une série de références bibliographiques.

Note remise et acceptée le 23 septembre 1997.

\section{Références bibliographiques}

[1] Buckholtz J. D., 1966 A characterization of the exponential series, Amer. Math. Monthly, 73, p. 121-123.

[2] Buckholtz J. D. et Shaw J. K., 1972. Zeros of partial sums and remainders of power series, Trans. Amer. Math. Soc., 166 , p. $269-284$

[3] Dieudonné J., 1935. Sur les zéros des polynômes-sections de e , Bull. Soc. Matl. France, 70, p. 333-351.

[4] Edrei A., Saff E. B. et Varga R. S., 1983. Zeros of sections of power series, Lecture Notes in Math., 1002, p. 1-115.

[5] Ganelius T., 1963. The zeroes of partial sums of power series. Duke Math. J., 30, p. 533-540.

[6] Goldberg A. A. et Ostrovskii I. V., 1970. Value distribution of meromorphic functions, Nauka, Moscow, Russian.

[7] Hedstrom G. W. et Korevaar J., 1963. The zeros of the partial sums of certain small entire functions, Duke Math. J., 30, p. 519-532.

[8] Koosis P., 1988. The logarithmic integral, I, Cambridge University Press, Cambridge.

[9] Nevanlinna R., 1970. Analytic functions, Springer, Berlin.

[10] Pólya G., 1913. Über die Annäherung durch Polynome mit lauter reelle Wurzeln, Rend. Circ. Math. Palermo, 36, p. $279-295$.

[1!] Pólya G. et Szegö G., 1976. Problems and theorems in Antlysis, vol. 2, Springer, Berlin.

[12] Yildirim C. Y., 1994. On the tails of the exponential series, Canad. Math. Bull., 37, p. 278-286. 